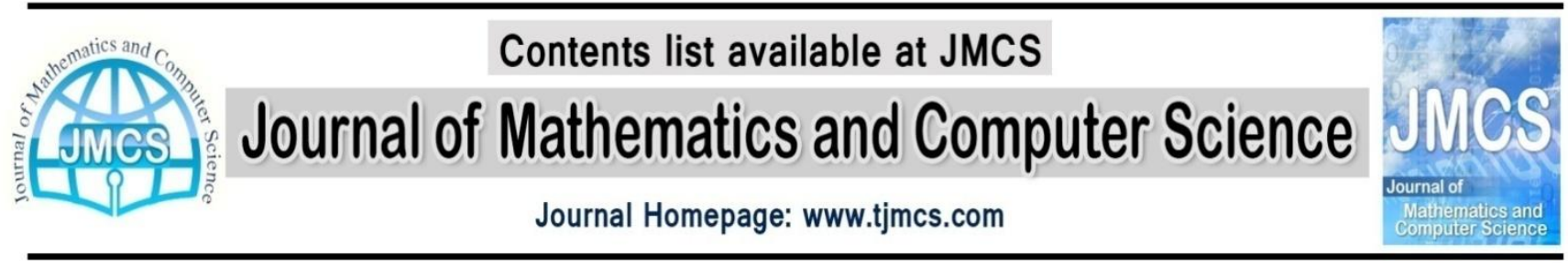

\title{
Cyclic Edge Extensions - Self-centered Graphs
}

\author{
Medha Itagi Huilgol \\ Department of Mathematics, Bangalore \\ University, Central College Campus, \\ Bangalore, Karnataka, India \\ medha@bub.ernet.in
}

\author{
Chitra Ramaprakash \\ Department of Mathematics Bangalore \\ University, Central College Campus, \\ Bangalore, Karnataka, India \\ r.chitraprakash@gmail.com
}

Article history:

Received March 2014

Accepted April 2014

Available online April 2014

\begin{abstract}
The eccentricity $e(u)$ of a vertex $u$ is the maximum distance of $u$ to any other vertex of $G$. The maximum and the minimum eccentricity among the vertices of a graph $G$ are known as the diameter and the radius of $G$ respectively. If they are equal then the graph is said to be a self - centered graph. Edge addition lextension to a graph either retains or changes the parameter of a graph, under consideration. In this paper mainly, we consider edge extension for cycles, with respect to the self-centeredness(of cycles), that is, after an edge set is added to a self centered graph the resultant graph is also a self-centered graph. Also, we have other structural results for graphs with edge -extensions.
\end{abstract}

Keywords: Self centered graphs, Edge extension graphs, reduced radius, reduced diameter of cycles, Iterations of cycles and paths.

\section{INTRODUCTION.}

Unless mentioned otherwise, for terminology and notation the reader may refer Buckley and Harary [7], new ones will be introduced as and when found necessary.

In this paper, by a graph $\mathrm{G}$, we mean a simple, undirected, connected graph without self-loops. The order and size are respectively the number of vertices denoted by $p$ and the number of edges denoted by $q$.

The distance $d(u, v)$ between any two vertices $u$ and $v$, of $G$, is the length of the shortest path between $u$ and $v$. The eccentricity $e(u)$ of a vertex $u$ is the distance to a farthest vertex from $u$. The maximum and the 
minimum eccentricity amongst the vertices of $\mathrm{G}$ are respectively called the diameter, diam $(G)$ and radius, $\operatorname{rad}(G)$. If $\operatorname{diam}(G)=\operatorname{rad}(G)$, then the graph $G$ is said to be a self-centered graph. If $\operatorname{dist}(u, v)=e(u),(v \neq u)$ then we say that $v$ is an eccentric vertex of $u$.

Harary [8], introduced the concept of changing and unchanging of a graphical invariant $i$, with interest in determining those for which $i(G-v)=i(G)$ and $i(G-v) \neq i(G)$ for all vertices $v$ of $G$, $i(G-e)=i(G)$ and $i(G-e) \neq i(G)$ for all edges $e$ of $G$ and $i(G+e)=i(G)$ and $i(G+e) \neq$ $i(G)$ for all edges $e$ of $\overline{\mathrm{G}}$, the compliment of $\mathrm{G}$. These concepts have been studied quite well for several invariants by Dutton et al. [9],Brigham et al.[15], [16],Harary [17],Lawson et al. [18], Medidi [19], Walikar et al.[11],[12],[13],[14] and Akram [4],[5]. Usually, these kind of studies reflect the variation of a parameter due to vertex or edge removal or edge addition, which find their applications in network analysis as they measure the results of link or equipment failure or network enhancement.

Janakiraman et al. [10] developed algorithms for constructing self-centered graphs from trees and connected graphs by adding edges. The authors defined a new concept called center number of a graph, denoted by $s c_{r}(G)$, which gives the minimum number of edges that can be added to a graph $G$ to obtain a self-centered graph.

Akram [4] studied the addition of edges by introducing the concepts of edge extension set of graphs, edge extensible class of graphs and the edge extensibility number of a graph. He defined a non -empty set $\mathrm{S}$ of edges as edge - extension set, such that every edge in $S$ joins two non-adjacent vertices in $G$. And

$G+S$, the graph after adding $S$ to it is called the edge extension graph.

\section{Definition 1 [4]}

Let $\tau$ be a class of graphs satisfying certain property. Then $\tau$ is called edge extensible class, if for every graph $G \in \tau, G$ is complete, or there exists an extension edge $e$ such that $G+e \in \tau$.

\section{Definition 2 [4]}

Let $G$ be a non-trivial simple graph (not complete). The simple graph obtained from $G$ by adding a nonempty set of edges $S$ such that every edge in $S$ joins two non-adjacent vertices in $G$ is called edge extension graph, and is denoted by $G+S, S$ is called the edge extension set. In particular, if $S$ consists of a single element $e$, then $e$ is called the extension edge, and the graph is denoted by $G+e$.

We can see that the graph $G+S$ has the vertex set and the edge set as follows. $V(G+S)=V(G)$ and $E(G+S)=E(G) \cup S$.

\section{Definition 3 [4]}

Let $\tau$ be a class of graphs with certain property and $G \epsilon \tau$ be non-trivial. The edge extensibility number of $G$ with respect to $\tau$ is the smallest positive integer $\mathrm{m}$, if exists, such that there exists an edge extension set $S$ of cardinality ' $m$ ' in such a way, the graph $G+S \epsilon \tau$. We write $m=\operatorname{ext}_{\tau}(G)$. If such a number does not exist for $G$, then we say that the corresponding edge extensibility number is $\infty$.

It is easy to note that the class of connected graphs is edge extensible class, but not regular graphs . On the other hand a tree with respect to the class of trees $\tau$ has extensibility number $\infty$. Akram [4],[5] has proved various results on extensibility of graphs and digraphs. Also, we can find results related to extension number of class of graphs viz., regular graphs, Eulerian graphs.

In this paper we consider self centered graphs as the collection $\tau$ and obtain edge extensibility number of some self centered graphs. Since cycles are the minimum sized self centered graphs, we find out 
$\operatorname{ext}_{\tau}(\mathrm{G})=m$.

It is clear that $m \neq 1$ for $\tau=C_{p}$. Hence, it is interesting and challenging to find the $\operatorname{ext}_{\tau}(C p)$.

\section{Edge extension for cycles}

In this section we consider edge extensions for cycles. As stated above it is clear that $m \neq 1$, with $m=\operatorname{ext}_{\tau}\left(C_{p}\right)$ where $\tau$ is the class of self-centered graphs. When an edge (set) is added to a cycle we see that it does not remain a self-centered graph. So the first result discusses the minimum number of edges required to be added to a cycle such that the resulting graph is a self centered graph. And, we denote $\mathrm{m}_{\mathrm{i}}=e x t_{\tau}\left(\mathrm{C}_{\mathrm{p}}\right)$ where $i$ denotes the amount by which the radius(diameter) of the cycle $C_{p}$ is reduced.

\section{Lemma 2.1}

Let $C_{p}$ be the class of cycles of length (order) $p$. Let $\tau$ be the set of self-centered graphs. Then,

$$
m_{1}=\operatorname{ext}_{\tau}\left(C_{p}\right)=\left\{\begin{array}{l}
3, \text { if } p \text { is even and } p \geq 12, \\
4, \text { if } p \text { is odd and } p \geq 11, \\
2, \text { when } p=4,8,9,10 \\
5, \text { when } p=5 \\
3, \text { when } p=6,7
\end{array}\right.
$$

Proof: Label the vertices of the cycle as $u_{1}, u_{2}, u_{3} \ldots u_{p}$.

For $p \geq 12$, with $p$ even, join $u_{1} u_{3}, u_{1} u_{p-1}$ and $u_{2} u_{4}$ so that the resultant graph is a self-centered graph of radius $(p / 2)-1$

For $p \geq 11$, with $p$ odd, join $u_{1} u_{3}, u_{1} u_{p-1}, u_{2} u_{4}$ and the fourth edge from $u_{p+1 / 2} u_{p+3 / 2}$, to get a self centered graph with reduced diameter, by one.

For $C_{4}$ and $C_{5}$ it is clear that $\operatorname{ext}_{\tau}\left(C_{4}\right)=2$ and $\operatorname{ext}_{\tau}\left(C_{5}\right)=5$ respectively, as they result into $K_{4}$ and $K_{5}$ on addition of edges.

For $C_{6}, \operatorname{ext}_{\tau}\left(C_{6}\right)=3$ and for $C_{7}, \operatorname{ext}_{\tau}\left(C_{7}\right)=3$.

And for $C_{8}, C_{9}, C_{10}$, the extension number is 2 as we can add the edges $u_{1} u_{3}$ and $u_{1} u_{p-1}$.

Hence the proof.

\section{Lemma 2.2}

Let $C_{p}$ denote the class of cycles of length (order) $p$, with $p \geq 11$. Then,

$$
m_{2}=\operatorname{ext}_{\tau}\left(C_{p}\right)=\left\{\begin{array}{l}
4, \text { if } p \text { is even and } p \geq 10, \\
5, \text { if } p \text { is odd and } p \geq 11, \\
7, \text { when } p=7, \\
4, \text { when } p=8, \\
9, \text { when } p=9 .
\end{array}\right.
$$

Proof: Label the vertices of the cycle as $u_{1}, u_{2}, u_{3}, \ldots . . u_{p}$.

For $p \geq 10$, with $p$ even, join the edges $u_{1} u_{3}, u_{1} u_{p-1}, u_{3} u_{6}$ and $u_{5} u_{8}$, to get a graph $G$ whose diameter is $t w o$ less than that of $C_{p}$.

For $p \geq 11$, with $p$ odd, join the edges $u_{1} u_{3}, u_{1} u_{p-1}, u_{p} u_{p-3}, u_{3} u_{6}$ and $u_{5} u_{8}$.

For $p=7$, adding one more $C_{7}$ to the existing one reduces the diameter by 2 . For $p=8$, adding 4 edges 
to each of their eccentric nodes reduces the diameter by 2 . For $p=9$, adding 9 more edges (one more $\mathrm{C}_{9}$ ) reduces the diameter by 2 . Hence the proof.

\section{Lemma 2.3}

Let $C_{p}$ denote the class of cycles with $p \geq 14$. Then, $m_{3}=\operatorname{ext}_{\tau}\left(C_{p}\right)=\left\{\begin{array}{l}6, \text { if } p \text { is even and } p \geq 14, \\ 8, \text { if } p \text { is odd and } p \geq 15,\end{array}\right.$

Proof: Label the vertices of the cycle $C_{p}$ as $u_{1}, u_{2}, u 3, \ldots . . u_{p}$.

If $p$ is even, with $p \geq 14$, then join the edges $u_{1} u_{3}, u_{3} u_{6}, u_{5} u_{8}, u_{1} u_{p-1}, u_{p-1} u_{p-4}$ and $u_{p-3} u_{p-6}$, so that the resultant graph has its diameter reduced by 3 .

If $p$ is $o d d$, with $p \geq 15$, then join $u_{1} u_{3}, u_{2} u_{4}, u_{3} u_{6}, u_{5} u_{8}, u_{1} u_{p-1}, u_{p} u_{p-3}, u_{p-2} u_{p-5}$ and $u_{p-4} u_{p-7}$ to obtain the required result. Hence the proof.

Similarly we can add edges to the cycle to reduce the radius/diameter until we get a complete graph. The above results give reduction of eccentricity of each vertex to be reduced by one, two or three. As generalization seems difficult, to find extension number for cycles, to be in class of self-centered graphs, the above results help us to measure the number of edges to be added.

In the next results we do not add single edge, instead, we add paths, but the resultant graph is a selfcentered graph. This way of approach is motivated by Buckley [6] in which he considered graphs under edge operation. Akira Saito et.al [1], [2], and [3] had considered properties of cycles of particular length. We combine both these approaches in the coming results.

\section{Definition 4[6]}

If $a \geq 4$, then $C_{a}{ }_{s} P_{b}$ consists of the graph formed from $C_{a}$ by joining two vertices $u$ and $v$ of $C_{a}$ at distance $b$ from one another by $s$ additional paths of length $b(b>1)$.

\section{Definition 5[6]}

If $a \geq 4$ and $1<d<b$, then $C_{a}{ }_{s} P_{b} * P_{d}$ is the graph formed from $C_{a}{ }_{s} P_{b}$ by joining the vertex $u$ to a vertex $w$ in $C_{a}$ at distance $d$ from $u$ by an additional path of length $d$.

\section{Lemma 2.4}

Let $C_{p}$ be a cycle of odd length, where $p \geq 7$. Then a path of length $P_{p}$ concatenated with two eccentric vertices in a cycle results in a self-centered graph.

Proof: Case (1) Consider a cycle $C_{7+4 k}$, which is of length - 7 modulo 4 where $k=0,1,2,3 \ldots$

Let $P_{2 p}$ be a path, where $p \in Z$ and $p \geq 2$.

On concatenating one end vertex of the path to any vertex say $u$ of $C_{p}$ and the other end vertex (of the path) to the eccentric vertex of $u$, say $v$, results in a self-centered graph. The length of the path varies depending on the radius of the cycle. Hence the path length $2 p$ is one less or two less than the radius of the cycle. 
Similarly, we can prove for the below two cases, by concatenating the specified path with a vertex and its eccentric vertex.

Case (2) Consider a cycle $C_{9+4 k}$, which is of length - 9 modulo 4 where $k=0,1,2,3 \ldots$ Let $P_{2 p+1}$ be a path, where $p \in Z$ and $p \geq 2$.

Case(3) Consider a cycle $C_{4+2 k}$, which is of length 4 modulo 2 where $k=0,1,2,3 \ldots$ Let $P_{p}$ be a path, where $m \in Z$ and $m=\operatorname{rad}\left(C_{p}-1\right)$ or $m=\operatorname{rad}\left(C_{p}-2\right)$ or $m=\operatorname{rad}\left(C_{p}\right)$.

Hence the proof.

\section{Remark 1}

A path of length $P_{2}$ can be added to $C_{7}$ and a path of length $P_{3}$ can be added to $C_{11}$ to obtain a self centered graph. A path of length $P_{2}$ and $P_{3}$ can be added to $C_{9}$ and a path of length $P_{4}$ can be added to $C_{13}$ to obtain a self centered graph.

In the next part we find the number of iterations, required for a cycle and a path to become a complete graph, using the concept of powers and the following three algorithms give the same.

\section{Algorithm 2.1}

In this algorithm we find the number of iterations required for cycle to be a complete graph.

Let $C_{p}$ be a cycle of length $p$.

STEP $1:$ Input the cycle length $p$.

STEP 2 : Find the eccentricity of the given cycle by using $e=p / 2$ if $\mathrm{n}$ is even or $e=$ floor of [p/2].

STEP 3 : If $e>1$, then increase the iteration. Next we start adding edges for the next iteration such that the distance between any two vertices is less than or equal to the iterated power. This is done for all the vertices of the cycle.

STEP 4 : Again checking for the eccentricity of all the nodes. If $e_{n e w}=1$, then GOTO STEP 6 else GOTO STEP 3.

STEP 5 : Print the iteration number.

STEP 6 : Print the number of iterations to get a complete graph.

STEP 7 : STOP.

\section{Algorithm 2.2}

In this algorithm we find the number of iterations required for path to be a complete graph.

Let $P p$ be a path on $p+1$ vertices.

STEP 1 : Input the path length.

STEP2 : Find the radius (minimum eccentricity) and the diameter (maximum eccentricity). The radius is denoted by $e_{\min }=a$ and the diameter is denoted by $e_{\max }=b$.

STEP 3 : If $e_{\min }>1$ and $e_{\max }>1$, increase the power else GOTO STEP 7

STEP 4 : Add edges to the consecutive node whose length $\leq$ iterated power.

STEP 5 : Find the new eccentricities, $e_{\text {new_min }}=a / 2$ and $e_{\text {new-max }}=b / 2$

STEP 6 : If $a / 2=1$ and $b / 2=1$, GOTO STEP 7 else GOTO STEP 3 . 
STEP 7 : Print the number of iterations.

STEP 8 : STOP.

\section{Algorithm 2.3}

In this algorithm we find the iteration number for cycles to be self-centered by adding edges.

Let $C_{p}$ be a cycle of length $p$.

STEP $1:$ Input the cycle length $p$.

STEP 2 : Find the eccentricity of the given cycle by using $e=p / 2$ if $p$ is even or $e=$ floor of $[p / 2]$, if $p$ is odd.

STEP 3 : ' $p$ ' denotes the length $=p=$ edges .

STEP 4 : Input the eccentricity reduction value.

STEP 5 : From the first vertex start adding edges one by one such that the eccentricity of the other vertices remain the same as inputted by the user.

STEP 6 : Perform STEP 5 until all the vertices have the same eccentricity.

STEP 7 : Check the eccentricity of the $2^{\text {nd }}, 3^{\text {rd }}$ and so on up to the $\mathrm{p}^{\text {th }}$ vertex. Check the eccentricity of all the vertices. If they are same GOTO STEP- 8 or GOTO STEP 5.

STEP 8 : Output List of available vertices.

STEP 9 : Output list of edges added and the number of edges added.

STEP 10 : Output list of Invalid nodes where the edge addition is not possible.

STEP 11 : STOP.

\section{CONCLUSION}

Characterization on the number of edges to be added to a general graph seems to be difficult at this point of time. Hence, particular cases give insight about the edge additions to retain a particular property. The results discussed in this paper deal with additions done to a cycle to retain its self centeredness.

\section{REFERENCES}

\section{Journal Articles}

[1] Akira Saito, Cycles of length 2 modulo 3 in graphs, Discrete Mathematics, 101(1992) 285-289.

[2] Akira Saito, Gantao Chen, Graphs with a cycle of length divisible by three, Journal of Combinatorial theory,60(1994),No.2, 277-292.

[3] Akira Saito, Linda Lesniak, Nathaniel Dean, Cycles of length 0 modulo 4 in graphs, Discrete Mathematics, 121(1993) 37-49.

[4] Akram B. Attar, Edge Extension of Graphs and Digraphs, The Journal of Mathematics and Computer Science, 3(2011), No.1, $1-10$.

[5] Akram B. Attar, Extensibility of Graphs, Journal of Applied Mathematics, Islamic Azad University of Lahijan, 6(2009),

No.21,1-9.

[6] F. Buckley, Self-centered graphs with a given radius, Congressus Numerantium, 23(1979) 211- 215.

[8] F. Harary, Changing and Unchanging radius of the graph, Linear Algebra and its Applications, 217(1995) 67-82.

[9] Ronald D. Dutton, Sirisha R Medidi and Robert C Brigham, Changing and Unchanging of the Radius of a Graph, Linear Algebra and its Applications, 217(1995),67-82.

[10] T.N. Janakiraman, M. Bhanumathi and S. Muthammai, Self - centered super graph of a graph and center number of a graph, Ars Combinatorica, 87(2008) 271-290.

[11] H.B. Walikar, F. Buckley, M.K. Itagi, Radius - edge - invariant and diameter - edge- invariant graphs, Discrete Mathematics , 272(1)(2001) 119-126. 
[12] H.B. Walikar, F. Buckley, M.K. Itagi, Diameter essential edges in a graph, Discrete Mathematics, 259(2002) 211-225.

[13] H.B. Walikar, F. Buckley, M.K. Itagi, Radius essential edges in a graph, Journal of Combinatorial Mathematics and Combinatorial Computing, 53(2005) 209-220.

[14] H.B. Walikar, F. Buckley, Medha Itagi Huilgol, Diameter vital edges in a graph, Aeqationes Mathematicae , 82(2011) 201-

211.

[15] R.C. Brigham and R.D. Dutton, An Extremal Problem for Edge Domination Insensitive Graphs, Discrete Appl. Math , 20(1988) 113-125.

[16] R.C. Brigham and R.D. Dutton, Changing and Unchanging Invariants: The Edge Clique Cover Number, Congr. Numer: , 70(1990) 145-152.

[17] F. Harary, Changing and Unchanging Invariants for Graphs, Bull. Malaysian Math. Soc:, 5(1982) 73-78.

[18] L. M. Lawson and T.W. Haynes, Changing and Unchanging of the Node Covering Number of a Graph, Congr. Numer:, 77(1991) 157-162.

\section{Books}

[7] F. Buckley, F.Harary, "Distance in Graphs”, Addison Wesley,1990.

\section{Thesis Reference}

[19] S.Medidi, The Effect of Node Deletions, Edge Additions and Edge Deletions on Graphs: Node Connectivity, Edge Connectivity, Diameter, and Radius, Masters Project, Dept. of Computer Science, Univ. of Central Florida, 1992. 